\title{
Update on the ESCEO recommendation for the conduct of clinical trials for drugs aiming at the treatment of sarcopenia in older adults
}

\author{
Jean-Yves Reginster ${ }^{1} \cdot$ Charlotte Beaudart $^{1}$ (1) - Nasser Al-Daghri' ${ }^{2} \cdot$ Bernard Avouac $^{3}$. Jürgen Bauer ${ }^{4}$. \\ Nathalie Bere ${ }^{5}$. Olivier Bruyère ${ }^{1}$. Francesca Cerreta ${ }^{5} \cdot$ Matteo Cesari $^{6,7} \cdot$ Mario Miguel Rosa $^{8} \cdot$ Cyrus Cooper $^{9}$. \\ Alfonso J. Cruz Jentoft ${ }^{10}$. Elaine Dennison ${ }^{9}$. Anton Geerinck ${ }^{1}$. Evelien Gielen ${ }^{11,24}$. Francesco Landi $^{12}$. \\ Andrea Laslop ${ }^{13}$. Stefania Maggi ${ }^{14}$. María Concepción Prieto Yerro ${ }^{15}$. René Rizzoli ${ }^{16}$. Hildrun Sundseth ${ }^{17}$. \\ Cornel Sieber ${ }^{18,25} \cdot$ Andrea Trombetti $^{16} \cdot$ Bruno Vellas $^{19} \cdot$ Nicola Veronese $^{20} \cdot$ Marjolein Visser $^{21} \cdot$ Mila Vlaskovska $^{22}$. \\ Roger A. Fielding ${ }^{23}$
}

Received: 5 June 2020 / Accepted: 16 July 2020 / Published online: 31 July 2020

(c) The Author(s) 2020

\begin{abstract}
Background In 2016, an expert working group was convened under the auspices of the European Society for Clinical and Economic Aspects of Osteoporosis and Osteoarthritis (ESCEO) and formulated consensus recommendations for the conduct of clinical trials for drugs to prevent or treat sarcopenia.

Aims The objective of the current paper is to provide a 2020 update of the previous recommendations in accordance with the evidence that has become available since our original recommendations.

Methods This paper is based on literature reviews performed by members of the ESCEO working group and followed up with face to face meetings organized for the whole group to make amendments and discuss further recommendations.

Results The randomized placebo-controlled double-blind parallel-arm drug clinical trials should be the design of choice for both phase II and III trials. Treatment and follow-up should run at least 6 months for phase II and 12 months for phase III trials. Overall physical activity, nutrition, co-prescriptions and comorbidity should be recorded. Participants in these trials should be at least 70-years-old and present with a combination of low muscle strength and low physical performance. Severely malnourished individuals, as well as bedridden patients, patients with extremely limited mobility or individuals with physical limitations clearly attributable to the direct effect of a specific disease, should be excluded. Multiple outcomes are proposed for phase II trials, including, as example, physical performance, muscle strength and mass, muscle metabolism and muscle-bone interaction. For phase III trials, we recommend a co-primary endpoint of a measure of functional performance and a Patient Reported Outcome Measure.

Conclusion The working group has formulated consensus recommendations on specific aspects of trial design, and in doing so hopes to contribute to an improvement of the methodological robustness and comparability of clinical trials. Standardization of designs and outcomes would advance the field by allowing better comparison across studies, including performing individual patient-data meta-analyses, and different pro-myogenic therapies.
\end{abstract}

Keywords Clinical trial $\cdot$ Sarcopenia $\cdot$ Guidelines $\cdot$ Recommendations $\cdot$ Drug registration $\cdot$ Treatment

\section{Introduction}

In 2016, a panel of experts from different disciplines reviewed and discussed the evidence available at the time and formulated consensus recommendations for the conduct

Charlotte Beaudart

c.beaudart@uliege.be

Extended author information available on the last page of the article of clinical trials for drugs to prevent or treat sarcopenia, under the auspices of the European Society for Clinical and Economic Aspects of Osteoporosis and Osteoarthritis (ESCEO) [1]. In the intervening years, important strides have been made in the field of sarcopenia, notably with regard to diagnostic criteria and its epidemiological characteristics [2]. The range of pharmacological therapies under investigation has expanded, although the earlier flurry of pharmacological therapies has subsided. However, among 
the pharmacological interventions that have been investigated few have shown positive results [3], which has led researchers to ask whether we missed a drug because we did not design the right trial for it. Both the absence of shared procedures and outcomes between clinical trials, differences in sample selection, as well as the uncertainty about the required duration of follow-up may have impacted results of previous clinical trials in sarcopenia. Furthermore, the current literature is confusing, with studies using diverse inclusion/exclusion criteria, treatment durations, concomitant therapies (e.g., exercise, nutrition interventions), and study outcome measures, complicating comparison of results [4].

In the 2016 recommendations, the working group members highlighted that a crucial next step in the evolution of sarcopenia research would be to find an agreement on an operational definition for sarcopenia, with accepted thresholds for abnormal muscle mass and muscle function [1]. In recent years, consensus has consolidated upon three consensus definitions, one European [European Working Group on Sarcopenia in Older People (EWGSOP)], one Asian [Asian Working Group on Sarcopenia (AWGS)] and one American [Sarcopenia Definitions and Outcome Consortium (SDOC)] $[2,5,6]$. The EWGSOP published its revised consensus statement in 2019, wherein they refined their previous diagnostic criteria and proposed an algorithm for case-finding. This group defined age-related sarcopenia, as "a progressive and generalized skeletal muscle disorder that is associated with increased likelihood of adverse outcomes including falls, fractures, physical disability and mortality" [2]. The diagnosis of sarcopenia in the EWGSOP2 and AWGS definitions established through a combination of low muscle mass and low muscle function (strength and/ or physical performance), but cut-off values and proposed tests and instruments differ between them $[2,5]$. The recent definition from the SDOC [6] takes a different approach to the diagnosis of sarcopenia. An international expert panel, guided by findings from 18 studies and literature reviews, voted on 13 position statements on grip strength, lean mass measured by Dual Energy X-ray absorptiometry (DXA), gait speed, and two summary statements. The panel strongly agreed that both low grip strength and low usual gait speed should be included in the definition of sarcopenia, and that these two indicators independently predict adverse outcomes in community-dwelling older people [6]. Although most efforts have been focused on age-related sarcopenia, also called primary sarcopenia, there is increasing awareness of the different clinical situations in which sarcopenia can present in an acute form, such as bedbound patients in hospital settings. Secondary sarcopenia occurs when other causal factors (in combination with or besides ageing) are at the source of the observed muscle wasting. Among these factors are comorbidities such as organ failure, inflammatory diseases, cancer and endocrine diseases; malnutrition, possibly due to gastrointestinal disorders, anorexia or psychosocial disorders; and inactivity due to a multitude of reasons [7].

Since 2016, age-related sarcopenia has been recognized as a muscle disease through an ICD-10-Clinical Modification code (M62.84) [8]. This code allows for sarcopenia to be recognized as a reportable condition by the US Food and Drug Administration (FDA) and the European Medicines Agency (EMA), and provides an additional argument for increased investment by industry and non-profit organizations into pharmacological interventions designed to improve sarcopenia-related outcomes [9].

In the past few years, a number of pharmacological interventions have been studied in clinical trials to understand whether they might prove to be effective in reducing or reversing the loss of muscle mass, muscle strength and physical function that characterizes sarcopenia $[10,11]$. Although a pharmacological agent has not been authorized yet, several pharmacologic agents have been reported to be under investigation. Rooks and Roubenoff provided an overview of the pharmacological approaches as of the end of 2018, detailing the different mechanisms of action such as the selective androgen receptor modulators (SARMS) and drugs that target the myostatin activin pathway. They list selective androgen receptor modulators, activin receptor agonists, myostatin or activin inhibitors fast skeletal muscle troponin activators and the Mas-receptor in the renin-angiotensin system [12]. In April 2020, a search on clinicaltrials.gov database for all clinical trials that listed sarcopenia as one target condition, yielded 461 study protocols. After manual selection, 44 interventional trials studying pharmacological interventions were withheld, the details of which can be found in appendix 1 .

A second expert working group composed of clinicians, researchers, representatives of the regulatory bodies and a patient advocate was convened in February of 2020 under the auspices of ESCEO. The present paper provides an update of the previous recommendations in accordance with the evidence that has become available in the meantime, to stimulate the debate about the design of phase II and phase III clinical trials in sarcopenia taking into account knowledge obtained in the last years.

\section{Methods}

In light of the evolution in pharmacological treatment options since 2016, and the need to link the current academic and regulatory perspectives, a second expert working group was convened in February of 2020 under the direction of ESCEO. This group assembled clinicians and researchers, as well as representatives of the regulatory bodies and a patient advocate. Certain members of the core writing group were asked to review the literature and/or to present the current 
state of the art on the following topics: (1) current status of sarcopenia drug development (FC); (2) did clinical trials in sarcopenia fail because of inappropriate design? (RF); (3) the impact of the EWGSOP2 sarcopenia definition on the selection of target populations for phase II and III trials (AC-J); (4) the identification of secondary endpoints (MC and FL); (5) patient-reported outcome measures in sarcopenia (CB); and (6) specific design aspects for clinical trials in sarcopenia (JB). The core writing group participated in presentations and discussion rounds, and created the preliminary version of these recommendations. A larger consensus group was solicited to provide written comments via email on the preliminary recommendations, which ultimately led to the consensus described in this article.

\section{Results}

\section{Target population for pharmacological treatment RCTs in sarcopenia}

\section{Inclusion criteria}

Potential target populations that should be included in pharmaceutical trials in sarcopenia have already been discussed in past publications [1, 13-16]. Based on these previous publications, as well as on the experience acquired in the field of sarcopenia since and ESCEO expert's opinion, we advocate to recruit patients, both for phase II or phase III trials, fulfilling the following criteria:

- Sex: both

- Age: 70 years and older with no upper age limit. Even if traditionally, 65 has been taken as the entry point into old age, working patterns are shifting and, in Western countries, it is often 70 years that is now considered as a threshold for old age. Moreover, the purpose of a clinical trial is obviously to obtain a benefit for the participants; to evaluate whether an intervention is beneficial, sarcopenia-related impairments must therefore be present to a relevant degree in the included population. Considering that the prevalence of sarcopenia has been shown to increase with age, the ESCEO working group recommends the inclusion of individuals with a minimal age limit of 70 years [17, 18].

- Physical abilities:

o Able to walk, and preferably able to perform the 400-m walk test within 15 min and without sitting, leaning or the help of another person or walker. This inclusion criterion is important as many events results from deambulation (falls, fractures, pain) and may be of high relevance in the context of primary and secondary outcome measurements.

o Presenting a combination of a low grip strength + low physical performance.

We recommend identifying the target population through objective measures of muscle strength and physical performance. Participants could be enrolled in a trial if they present both a reduction of their muscle strength capacities and of their physical performance capacities $[6,16]$. This "sarcopenia risk profile" could optimize the probability to detect a meaningful difference between intervention and control group.

We acknowledge that muscle mass has been an important diagnostic criterion for sarcopenia. But, so far, low muscle mass has been shown to be less related to health-related outcomes, as compared with other sarcopenia criteria. Moreover, there are still important methodological issues with regard to the measurement of muscle mass. Therefore, interventions for sarcopenia should not be restricted to sarcopenic patients, thus not be limited to participants presenting with low muscle mass combined with low muscle strength. To enlarge inclusion criteria, it has been decided to leave out muscle mass as inclusion criterion (at least, to date), but we underline that this proposal only applies to inclusion criteria and not to outcome measurement.

The validated cut-offs proposed for the criteria low grip strength and low physical performance are available in Table 1. Any of the proposed tools could be used without restriction, depending on the logistic capacities of the recruitment centre. The most appropriate procedure of administration, equipment, performance (i.e., feasibility, reliability and floor/ ceiling effects) and well as the references range of each of these tools have already been described in a previous publication endorsed by ESCEO [19].

\section{Exclusion criteria}

- Nutritional status of all participants should be evaluated during the recruitment stage, and those who are severely malnourished should not be included in the trial. Malnutrition could be measured using one of the several available diagnostic tool; Mini-Nutritional assessment tool, European Society of Clinical Nutrition and Metabolism (ESPEN) criteria, Global Leadership Initiative of Malnutrition (GLIM) criteria, etc. [26-28], Indeed, malnutrition has been shown to influence muscle mass and is a strong predictor of sarcopenia. More- 
Table 1 Inclusion criteria related to muscle strength and physical performance

\begin{tabular}{lll}
\hline & Tool proposed & Cut-offs proposed \\
\hline Low muscle strength & Hydraulic handheld isometric dynamometer (i.e., & $\begin{array}{c}\text { Maximal value from three attempts for each } \\
\text { hands }[20]:<27 \mathrm{~kg} \text { for men; }<16 \mathrm{~kg} \text { for } \\
\text { women }[21]\end{array}$ \\
& JAMAR or similar) & $>15 \mathrm{~s}$ for five rises [22] \\
Low physical performance & Chair stand test & $\leq 8$ points [23] \\
& SPBB test & $\leq 0.8 \mathrm{~m} / \mathrm{s} \mathrm{[24]}$ \\
& Gait speed (4-m) & $\geq 20 \mathrm{~s} \mathrm{[25]}$ \\
\hline
\end{tabular}

$S P P B$ short physical performance battery

over, malnourished participants have been shown to be at higher risk of mortality over a short-term period [29, 30].

- Patients with acute immobility (i.e., post hip fracture or post-acute hospital admission) should be excluded.

- Patients suffering from specific advanced pathologies such as, for example, terminal cancer, severe renal diseases [e.g., Estimated Glomerular Filtration Rate (eGFR) < 30], chronic obstructive pulmonary diseases (COPD) requiring oxygen, etc. should not be included in the clinical trial.

- Factors that may affect conduct of the trial (e.g., physical limitations should not be clearly attributable to the direct effect of a specific disease other than sarcopenia). In particular, patients with a physical limitation clearly attributable to the direct effect of a specific disease other than sarcopenia should be excluded (e.g., patients with a diagnosis of dementia or score $<24$ on the Mini Mental State Examination (MMSE), patients with serious neurological, neuromuscular or orthopaedic conditions (e.g., Parkinson's disease), patients with thymic disorders (e.g., anxious or depressive syndrome)).

Considering the number of different pathways linked to the sarcopenia and the large heterogeneity of the older adult population, exclusion criteria recommended could depend on the target (age-related primary sarcopenia only, secondary sarcopenia only, or both), on mechanism of action of the drug/agent/therapy, and on the clinical setting. Research teams should consider these specificities before finalizing specific exclusion criteria.

The exclusion criteria are used to build more robust samples, reduce the rate of dropouts and enhance the effect of the intervention. However, having stringent exclusion criteria might lead to the predominant inclusion of people with primary sarcopenia. If the study is targeting another distinct population of patients, exclusion criteria might need be reconsidered and modified.

\section{Primary and secondary outcomes for pharmacological treatment RCTs in sarcopenia}

\section{Phase II studies}

Phase II trials are designed with two important objectives: 1/obtaining a "proof of concept" for the new entities, 2/ allowing a clear assessment of the effective dose-range to highlight the best dose to use in phase III trials within an economically reasonable time frame.

Different primary endpoints could be proposed in phase II studies. As a primary consideration, measures with a higher rate of change over time may reduce the followup duration and could therefore be chosen as a primary endpoint in phase II trials. However, since the objective of phase II clinical trials is to demonstrate the efficacy of the intervention, the study design might be more flexible since it is based on surrogates of the condition of interest. Table 2 displays all outcomes that we considered as applicable in phase II studies.

The choice of outcome to be used in phase II trial as primary endpoint - which is the responsibility of the applicant-should be done according to the hypothesized mode of action of the drug.

\section{Phase III studies}

Phase III studies are pivotal for the applicant to demonstrate that the currently investigated new chemical entity is safe and effective for the management of sarcopenia.

\section{Primary endpoint}

This outcome should be clinically relevant, highly responsive to treatment effects and methodologically robust. The primary outcome in phase III studies is used to calculate 
Table 2 List of outcomes that could be applicable in phase II studies (the final choice is the responsibility of the applicant)

\begin{tabular}{|c|c|}
\hline Proposed endpoint & Tool to measure the endpoint \\
\hline \multirow[t]{5}{*}{ Improvement of physical performance } & SPPB \\
\hline & Gait speed \\
\hline & 400-m walk test \\
\hline & TUG test \\
\hline & Chair stand test \\
\hline \multirow[t]{2}{*}{ Improvement of muscle strength } & Handgrip strength \\
\hline & Knee extensor strength \\
\hline \multirow[t]{5}{*}{ Increase of muscle mass } & CT-scan \\
\hline & MRI \\
\hline & DXA \\
\hline & Urinary D-3 creatine \\
\hline & Muscle biopsy \\
\hline \multirow[t]{4}{*}{ Improvement of muscle quality* } & CT-scan \\
\hline & MRI \\
\hline & MRS \\
\hline & Muscle biopsy \\
\hline Muscle metabolism & Biomarkers of muscle metabolism \\
\hline Muscle-bone interaction & $\begin{array}{l}\text { Biomarkers of muscle-bone interaction (e.g., Myostatin, Activin A, } \\
\text { amino terminal of type III procollagen peptide (P3NP), insulin-like } \\
\text { growth factor-1 (IGF-1) [31, 32]) }\end{array}$ \\
\hline
\end{tabular}

*For example, decrease in intramyocellular lipid accumulation and intermuscular adipose tissue, increase in muscle blood flow, change in fibre types and increase in skeletal muscle mitochondrial capacity

$M R S$ magnetic resonance spectroscopy, $S P P B$ short physical performance battery, $T U G$ timed Up and Go, CT-scan computerized tomography scan, $M R I$ magnetic resonance imaging

the necessary sample size and to inform the randomization procedures.

We recommend, for all phase III clinical trials in sarcopenia, the use of co-primary endpoints, combining a measure of physical performance with a Patient Reported Outcome Measure (PROM). With this proposal, we ensure to capture first an objective endpoint, by recommending a physical performance measure, for which a surrogate value for hard clinical endpoints such as mortality, hospitalisation, fractures and falls is already well established [33]. Second, the use of a PROM will allow to capture more subjective but equally important aspects of patient-relevant efficacy since the patient perspective is now recognized as key parameters in the evaluation of health interventions [34, 35]. There is an increasing emphasis on patient-centred research and PROMs are increasingly recognized by government regulatory agencies (e.g., FDA, EMA, etc.), clinicians and patients as valuable tools to be used in clinical trials to ensure the impact of a clinical intervention is comprehensively assessed [36]. The way of how patients perceive the benefit derived from an intervention could be captured by the use of PROM. A common issue of co-primary endpoints appears when the two co-endpoints respond differently to the treatment [37, 38]. However, combining an objective primary endpoint such as physical performance with a subjective well-being endpoint such as quality of life reduces the risk for this scenario. Indeed, previous studies have already highlighted the linear relationship between the improvement of physical performance and health-related quality of life in older adults $[39,40]$. This combination of physical performance and PRO is also likely to be relevant for Health Technology (reimbursement) assessment.

Physical performance is clinically relevant, considered as most important by patients themselves as compared with muscle strength and muscle mass, easy to implement in clinical settings and closely related to muscle health. Moreover, normative data and thresholds to define meaningful change are available for this outcome [41]. Among the different instruments available to measure physical performance, the 400-m walk test seems to best reflect autonomy [42]. Indeed, this distance is a common distance used to assess self-reported physical performance and believed to be required for independence with daily tasks. Moreover, inability to complete the $400-\mathrm{m}$ walk test is highly associated with negative health outcomes (mortality, cardiovascular event, disability) and higher healthcare costs [42-44]. Finally, previous published data in intervention studies using the 400-m walk test as primary outcome are available for the calculation of sample size of any new clinical trial [17, 18]. We recommend measuring the incidence of inability to 
perform the 400-m walk test (also called mobility disability) as primary endpoint. Other tests, such as the SPBB test, deeply correlated with physical performance as measured by the 400-m walk test could also be proposed [45]. Moreover, SPPB is also linked to more distal clinical endpoints such as falls, fractures, nursing home admission and mortality $[46,47]$.

There are several reasons why muscle mass and muscle strength are not considered to be appropriate candidates for a primary endpoint. First, the different tools available to measure muscle mass and strength are very heterogeneous and no proper and valid gold standard has categorically been defined. Second, there is a lack of meaningful thresholds to be adopted, especially to monitor changes over time. Finally, based on patient's preferences, muscle mass and strength seem to be of secondary importance compared to more comprehensive measures of functioning [48].

Regarding PROMs, currently, two different PROMs specific to sarcopenia are available: the Age-Related Muscle Loss questionnaire (ARMLQ), a PROM measuring the functional impact of reduced muscle strength from the patient perspective [49] and the SarQoL ${ }^{\circledR}$ questionnaire [50, 51], a health-related quality of life questionnaire for sarcopenia. So far, only the SarQoL ${ }^{\circledR}$ questionnaire has been validated with regard to the responsiveness. Geerinck et al. [40] reported that this specific instrument is more sensitive to change as compared with generic tools largely used as PROMs, such as the SF-36 or EQ-5D questionnaire. Publications [52] related to the $\mathrm{SarQoL}{ }^{\circledR}$ questionnaire also provide its standard error of measurement (2.65 points on a scale of $0-100$ points) and its smallest detectable change ( 7.35 points on a scale of 0-100 points), through the combination of nine cohort studies, which provides a high external validity and useful data for clinical trials. Finally, this tool is available in more than 27 languages, all translations being performed by following rigorous guidelines. Because the specific questionnaires are more precise and responsive to change compared with generic questionnaires, we recommend the use of those specific instruments in clinical trials on sarcopenia. To be able to obtain a comparison with other trials and a certain generalizability of data, it is possible to combine a generic tool with a specific tool and therefore, obtain a more accurate proxy of treatment efficacy. A third PROM is currently being validated for use in sarcopenia and may provide an interesting option in the future. The Patient-Reported Outcomes Measurement Information System (PROMIS ${ }^{\circledR}$ ) is a list of self-report measures covering multiple domains within physical, mental and social health. They have been developed as item banks, allowing for computerized adaptive testing, as well as the extraction of short form questionnaires [53]. Currently, a project funded by the Food and Drug Administration (1U01FD006887-01) is underway to certify the PROMIS measure of physical function as a clinical outcome assessment, and to investigate the specific context of use in which it could serve as a primary metric [54].

\section{Secondary endpoint}

Secondary endpoints in phase III studies are also necessary to determine the efficacy of a treatment and their results are expected to be consistent with the primary endpoints. Secondary outcomes are generally not considered in sample size calculation. Secondary variables are either supportive measurements related to the primary objective or measurements of effects related to the secondary objectives. Their pre-definition in the protocol is also important, as well as an explanation of their relative importance and roles in interpretation of trial results. Therefore, the statistical power to measure them is not necessarily ensured and results of secondary endpoints should be interpreted with caution. Even if, because of the reasons presented above, muscle strength and muscle mass cannot be used as primary endpoints, they are appropriate candidates for secondary endpoints. However, the limitations mentioned above regarding muscle strength and muscle mass still apply. Investigators need therefore to be cautious when using these measurements. For muscle strength, we recommend using the handgrip strength measure, because it is a highly feasible measure [55] that is part of the diagnosis of sarcopenia. The protocol of Roberts et al. [56] should be applied. Because different brands of devices could lead to different measures of strength [57], we also recommend the use of the hydraulic handheld JAMAR device (or similar) to be as standardized as possible. Unfortunately, so far, data on sensitivity of change in grip strength to interventions are still rather limited and inconsistent $[58,59]$. Only one study proposed a minimal change of $6 \mathrm{~kg}(13.2 \mathrm{lb})$ in older women to be considered as clinically significant [60]. However, several non-pharmacological intervention studies have shown an increase of muscle strength following resistance exercises combined or not with nutritional interventions, which highlight the potential sensitivity to change of this measure following an intervention $[14,61]$. With regard to muscle mass we recommend the use of Dual Energy X-ray absorptiometry as the current measure of choice for the assessment of fat-free mass and appendicular lean body mass, despite its well-known limited accuracy in the estimation of muscle mass and the expensive equipment. This measure has been widely used and validated reference ranges are available in the field of sarcopenia [2, 62]. CT-scans and MRI also constitute appropriate devices for the accurate measurement of muscle mass. Both tools are expensive and require certified personnel. There are other techniques, such as bioelectrical impedance analysis (BIA), ultrasound and anthropometric measures, but we presently discourage their use in a clinical trial as secondary endpoints because of their limited accuracy or insufficient published 
data on their validity [63-66]. At this moment more studies are needed before the relevance of D3-creatine for the assessment of muscle mass can be estimated on a sound basis. All possible primary endpoints proposed in phase II (Table 2) could also be proposed as secondary endpoints in phase III trials. This includes, as exploratory endpoints, markers of muscle quality, biomarkers of muscle metabolism and of muscle-bone interactions to support the mode of action of new chemical entities.

\section{Study design for pharmacological treatment RCTs in sarcopenia}

The study design that should be privileged is the randomized, placebo-controlled, parallel arm trial performed in a double-blind manner. Randomization procedures should be carefully performed with stratification when needed. Primary analyses should ideally be run on an intent-to-treat basis and should include all randomized participants who received at least one dose of treatment.

The intervention should generally be a single intervention. Multi-domain interventional trials, combining several approaches in one group compared with alternative combined interventions or a control group are not recommended because it may increase the difficulty to identify the intervention which is the most effective.

A complete protocol should be registered in a trial registry before participant recruitment starts. We also encourage online publication of open-access datasets for transparency and to encourage the future realisation of individual patient data (IPD) meta-analyses.

\section{Length of treatment, follow-up and time point assessment}

\section{Phase II}

Taking into account the annual rate of decline in physical performance, muscle mass and strength [67, 68], a recommendation of a minimum 6-month treatment and followup period was suggested upon for phase II trials. A shorter period might be acceptable depending on the mode of action.

\section{Phase III}

The members of the Working Group, who have a close interaction with the European regulatory authorities, feel that a minimum of 1 year of follow-up is necessary for safety issues. However, treatment studies with a longer duration are often confronted with higher numbers of dropouts which is especially relevant for older study populations. Based on this and to appreciate the offset of action, phase III clinical trial for sarcopenia may consist of a 1-year treatment period. Assessment of co-primary endpoints could be performed every 3 months. We encourage trials to use, as much as possible, similar time points for assessment to allow comparison between studies and to reduce the risk of introducing heterogeneity due to time-point assessment in meta-analyses and network meta-analyses.

\section{Comparator}

The comparator should be placebo along with standard of care.

Because exercises and/or nutrition could be defined as usual care, these aspects should be recorded and controlled and standardized (e.g., cross countries harmonization for multicentre studies) as much as possible and should be clearly defined in the protocol. This possibility of adding standard care in the protocol of intervention is offered, considering the length of the clinical trial and to be in accordance with the WHO recommendations for encouraging of physical activity in older people. Older adults could benefit from physical activity and nutrition, both interventions having been proven for the improvement of muscle strength and physical performance [14, 61].

\section{Nutrition/exercise/polypharmacy record}

Randomized controlled trials, despite being the best design for both Phase II and Phase III trials, would have to address numerous cofounders. Important exogenous confounders that formally require consideration are nutritional status, physical activity level, dietary pattern, comorbidities and coprescriptions. If possible, these confounding factors should be equally matched across treatment arms. Since despite randomisation, baseline characteristics may differ between the intervention and the control group, appropriate adjustments for these differences need to be considered in the main analyses. Some subgroups might be more sensitive to the intervention than the general population. It is the responsibility of the applicant to take this possibility into account.

Ageing is associated with anabolic resistance [69]. Factors such as protein intake, vitamin $\mathrm{D} /$ calcium, and the acid-base balance of the diet, play an important role in maintaining muscle mass and, muscle strength and physical performance [61, 70-75]. Poor nutritional status has therefore been recognised as one of the etiologic mechanisms contributing to sarcopenia [30]. Moreover, malnutrition is a major cause of adverse health consequences, such as impaired physical function, hospitalization, and mortality in older people and could therefore impact negatively the results of clinical trials on sarcopenia $[29,76]$. For these reasons, at baseline, nutritional status should be assessed and, as described in the exclusion criteria, severely malnourished 
participants should not be included in sarcopenia clinical trials. Besides, it is necessary to re-assess nutritional status over time during the trial, at least on each time point assessments. In addition, monitoring dietary intake during the trial is advised.

The positive impact of physical activity on muscle health is well recognized. Numerous studies have highlighted improvement of muscle mass, muscle strength and physical performance following exercises $[14,61,77-80]$. The level of physical activity within the included population is therefore an important confounding factor. We recommend recording physical activity using technological devices that may record all day activity, and not only exercises (e.g., by an inertial measurement unit, pedometers, connected watches, etc.), and, if not logistically possible, to document carefully the level of physical activity, at least on each time point assessment, through physical activity questionnaires that have been validated for use in older populations (e.g., Minnesota scale [81], IPAQ-E questionnaire, PASE [82], etc.).

The population of interest (e.g., older people suffering from muscle impairments) is a population at high risk of polypharmacy. For good clinical practice, poly-medication also needs to be carefully recorded at baseline and at each time point assessment, specifically in multi-morbid older patients with polypharmacy [83].

Finally, apart from recording major comorbidities at study entry, a measure of the global burden of comorbidities [e.g., Charlson comorbidity index or Cumulative Illness Rating Scale (CIRS-G)] may also be proposed. Thymic disorders, cognitive disorders and frailty status are also recommended to be recorded and considered as potential confounding factors.

Health events should also be recorder throughout all the study period. Among them, hospitalisations, falls, fractures, functional decline and institutionalization should be documented in each time point assessment. These health events should be considered as either as a marker of efficacy (i.e., their absence) either as an important confounder (e.g., long stay at the hospital for an infectious disease requiring a 6-week antibiotics treatment is supposed to have serious muscle consequences).

\section{Stratification}

Stratification in clinical trials consists of partitioning subjects and results into subgroups differenced by factors other than the treatment given. To consider as many confounders or discrepant levels of response in some subgroups, performing stratification could therefore be a solution. Any subgroup analysis in phase III trials should be pre-specified. It could be therefore possible to stratify results on different parameters: on the value of primary outcome at baseline (time recorded for the $400 \mathrm{~m}$ walk test), on gender, on age, on comorbidities, on frailty status at baseline and, finally, on the baseline value of the variable that is intervened on (e.g., with a intervention on protein metabolism, it is possible to stratify on baseline protein intake), etc.

\section{Rescue medications}

Given that no drugs are currently available, the issue of rescue medications is not applicable.

\section{Consensus}

The summary of the consensus is presented in Table 3 which includes all the recommended criteria for the conduct of any new phase II and phase III pharmacological trial in sarcopenia.

\section{Conclusion}

The present ESCEO working group was born out of concern that we may be overlooking a drug treatment for sarcopenia because we did not design the appropriate trial for this purpose. Much knowledge has been gathered in the last few years with regards to clinical trials investigating pharmaceutical interventions in sarcopenia, and the working group convened to discuss the lessons learned from recent clinical trials. The working group has formulated consensus recommendations on specific aspects of trial design, and in doing so hopes to contribute to an improvement of the methodological robustness and comparability of clinical trials, but also acknowledges that uncertainties remain. Sarcopenia remains an important challenge for ageing individuals, and it is therefore important to devote time and effort to the search for ways to prevent, slow down or treat muscle weakness. 
Table 3 Recommended criteria for the conduct of any new phase II or phase III pharmacological trial in sarcopenia

\begin{tabular}{|c|c|c|}
\hline & Phase II trials & Phase III trials \\
\hline Appropriate study design & $\mathrm{RCT}$, placebo-controlled double blind & RCT, placebo-controlled double blind \\
\hline \multirow[t]{3}{*}{ Inclusion criteria } & Age 70 and + & Age 70 and + \\
\hline & Able to walk & Able to walk \\
\hline & Low muscle strength + low physical performance & Low muscle strength + low physical performance \\
\hline \multirow[t]{4}{*}{ Exclusion criteria } & Severe malnutrition & Severe malnutrition \\
\hline & Acute immobility & Acute immobility \\
\hline & Specific advanced pathologies & Specific advanced pathologies \\
\hline & $\begin{array}{l}\text { Physical limitation attributable to a specific disease } \\
\text { other than sarcopenia }\end{array}$ & $\begin{array}{l}\text { Physical limitation attributable to a specific } \\
\text { disease other than sarcopenia }\end{array}$ \\
\hline Primary outcome & See list on Table 2 & $\begin{array}{l}\text { Co-primary endpoint: } 1 / \text { incidence of inability to } \\
\text { walk the } 400 \text {-m walk test }+2 / \text { PROM }\end{array}$ \\
\hline \multirow[t]{2}{*}{ Secondary outcome } & See list on Table 2 & Muscle strength (JAMAR dynamometer) \\
\hline & & Muscle mass (DXA) \\
\hline Length of treatment/follow-up & 6 months of treatment and follow-up & 1 year of treatment and follow-up \\
\hline Time point assessment & Every 3 months & Every 3 months but at least every 6 months \\
\hline Comparator & Placebo & Placebo \\
\hline Co-treatment & Standard care for both groups & Standard care for both groups \\
\hline \multirow[t]{5}{*}{ Confounding } & Nutritional status & Nutritional status \\
\hline & Physical activity & Physical activity \\
\hline & Co-prescriptions & Co-prescriptions \\
\hline & Comorbidities & Comorbidities \\
\hline & Health events & Health events \\
\hline \multirow[t]{6}{*}{ Stratification } & Value of primary outcome at baseline & Value of primary outcome at baseline \\
\hline & Gender & Gender \\
\hline & Age & Age \\
\hline & Comorbidities & Comorbidities \\
\hline & Frailty status & Frailty status \\
\hline & Variable intervened & Variable intervened \\
\hline Rescue medication & NA & NA \\
\hline
\end{tabular}

Author contributions JYR organized the meeting. JB, CB, FC, MC, ACJ, FL and RF performed the literature review. JYR, RF, CC and CB has drafted the manuscript. All authors have taken part in the discussion and meeting and have critically revised, and given inputs to the final manuscript.

Funding The ESCEO Working Group was funded by ESCEO. The ESCEO receives unrestricted education Grants to support its educational and scientific activities from non-governmental organisations, not-for-profit organisations, non-commercial or corporate partners. The choice of topics, participants, content and agenda of the Working Group as well as the writing, editing, submission and reviewing of the manuscript are under the sole responsibility of the ESCEO, without any influence from third parties.

\section{Compliance with ethical standards}

Conflict of interest The views expressed in this article are the personal views of the authors and may not be understood or quoted as being made on behalf of or reflecting the position of the agencies or organizations with which the authors are affiliated. C. Beaudart reports to be a shareholder of SARQOL SPRL, a spin-off of the University of Liege.
O. Bruyere reports grants or fees from AMGEN, APTISSEN, BIOPHYTIS, IBSA, MEDA, SANOFI, SERVIER, SMB, THERAMEX and UCB. He also is a shareholder of SARQOL SPRL, a spin-off of the University of Liege. E. Dennison reports honoraria from UCB and PFIZER. E. Gielen reports consultancy fees from UCB, ALEXION, and TAKEDA, and speaker's fees from AMGEN and TAKEDA. S. Magggi reports grants from SANOFI PASTEUR, MSD, GSK, PFIZER, TAKEDA through institution as organizer of meetings/congresses and as principal investigator of epidemiological studies, for taking part to advisory boards and expert meeting. J-Y. Reginster reports consulting fees or paid advisory boards (IBSA-GENEVRIER, MYLAN, RADIUS HEALTH, PIERRE FABRE, ECHOLIGHT, TEVA), lecture fees when speaking at the invitation of sponsor [IBSAGENEVRIER, MYLAN, CNIEL, DAIRY RESEARCH COUNCIL (DRC), TEVA], Grant Support from Industry (All through Institution) (IBSA-GENEVRIER, MYLAN, CNIEL, RADIUS HEALTH). He also is a shareholder of SARQOL SPRL, a spin-off of the University of Liege. N. Veronese reports personal fees from MYLAN, FIDIA, IBSA. Other authors reported no conflicts of interest.

Ethical approval Not applicable.

Informed consent Not applicable. 
Open Access This article is licensed under a Creative Commons Attribution 4.0 International License, which permits use, sharing, adaptation, distribution and reproduction in any medium or format, as long as you give appropriate credit to the original author(s) and the source, provide a link to the Creative Commons licence, and indicate if changes were made. The images or other third party material in this article are included in the article's Creative Commons licence, unless indicated otherwise in a credit line to the material. If material is not included in the article's Creative Commons licence and your intended use is not permitted by statutory regulation or exceeds the permitted use, you will need to obtain permission directly from the copyright holder. To view a copy of this licence, visit http://creativecommons.org/licenses/by/4.0/.

\section{Appendix}

See Table 4.

Table 4 Study protocols registered in the clinicaltrials.gov database on 22 April 2020 with sarcopenia listed as a target condition

\begin{tabular}{|c|c|c|c|c|c|}
\hline NCT number & Status & Conditions & Interventions & Start date & Completion date \\
\hline NCT04021706 & Recruiting & $\begin{array}{l}\text { Sarcopenia } \\
\text { Osteopenia }\end{array}$ & Anamorelin hydrochloride & $05-12-19$ & $01-11-21$ \\
\hline NCT03995251 & Recruiting & Liver cirrhosis & Testosterone & 04-07-19 & $30-06-20$ \\
\hline NCT03867357 & Recruiting & $\begin{array}{l}\text { Metastatic prostate cancer } \\
\text { Androgen deprivation therapy }\end{array}$ & GnRH agonist & $07-12-18$ & $31-08-21$ \\
\hline NCT03788252 & Active, not recruiting & Chronic kidney diseases & Renamezin & 23-11-18 & $01-12-20$ \\
\hline NCT03633279 & Recruiting & Liver cirrhoses & Branched chain amino acid & $22-06-18$ & $01-07-20$ \\
\hline NCT03452488 & Recruiting & $\begin{array}{l}\text { Sarcopenia } \\
\text { Gait disorders in old age } \\
\text { Muscle weakness }\end{array}$ & BIO101 & $24-05-18$ & $01-12-20$ \\
\hline NCT03248271 & Withdrawn & $\begin{array}{l}\text { Diabetes } \\
\text { Sarcopenia } \\
\text { Hypotension, orthostatic }\end{array}$ & Insulin Lispro & 01-10-17 & 01-10-19 \\
\hline NCT03119610 & Active, not recruiting & $\begin{array}{l}\text { Obesity } \\
\text { Sarcopenic obesity } \\
\text { Sarcopenia } \\
\text { Aging } \\
\text { Sedentary Lifestyle }\end{array}$ & Oxytocin & 22-09-17 & 01-12-19 \\
\hline NCT02938923 & Recruiting & $\begin{array}{l}\text { Hip fracture } \\
\text { Frailty } \\
\text { Sarcopenia }\end{array}$ & Testosterone & $15-09-17$ & $31-05-22$ \\
\hline NCT03054168 & Active, not recruiting & $\begin{array}{l}\text { Sarcopenia } \\
\text { Muscle hypotrophy } \\
\text { Muscle atrophy }\end{array}$ & Sustanon & $15-12-16$ & $15-02-19$ \\
\hline NCT01417364 & Withdrawn & Sarcopenia & Testosterone enanthate & $01-01-16$ & $01-12-17$ \\
\hline NCT02575235 & Unknown status & Sarcopenia & $\mathrm{CPC}$ & $01-10-15$ & 01-08-16 \\
\hline NCT02594579 & Unknown status & $\begin{array}{l}\text { Vitamin D deficiency } \\
\text { Sarcopenia } \\
\text { Critical illness }\end{array}$ & Vitamin D3 & $01-10-15$ & $01-12-16$ \\
\hline NCT02468674 & Completed & Sarcopenia & Bimagrumab & $22-07-15$ & $03-12-18$ \\
\hline NCT01550107 & Completed & Sarcopenia & Allopurinol & $01-02-15$ & $20-09-17$ \\
\hline NCT02333331 & Completed & Sarcopenia & Bimagrumab & $09-12-14$ & $28-06-18$ \\
\hline NCT02297997 & Unknown status & Sarcopenia & Cetylpyridinium chloride & $01-11-14$ & $01-11-15$ \\
\hline NCT02370745 & Completed & Sarcopenia & $\begin{array}{l}\text { GLP-1 } \\
\text { Insulin actrapid } \\
\text { GIP }\end{array}$ & $01-11-14$ & $01-03-18$ \\
\hline NCT02606279 & Terminated & $\begin{array}{l}\text { HIV } \\
\text { Aging } \\
\text { Sarcopenia } \\
\text { Angiotensin receptor antagonists }\end{array}$ & Valsartan & 01-07-14 & 04-08-16 \\
\hline
\end{tabular}


Table 4 (continued)

\begin{tabular}{|c|c|c|c|c|c|}
\hline NCT number & Status & Conditions & Interventions & Start date & Completion date \\
\hline NCT04354896 & Completed & $\begin{array}{l}\text { Subclinical hypothyroidism } \\
\text { Sarcopenia }\end{array}$ & Levothyroxine & $01-05-14$ & $04-05-18$ \\
\hline NCT01804049 & Completed & Prediabetes & Metformin & 08-04-14 & $31-08-18$ \\
\hline NCT03784495 & Completed & Sarcopenia & Melatonin & 01-01-14 & 01-06-15 \\
\hline NCT01963598 & Completed & Sarcopenia & REGN1033 & 01-11-13 & $01-02-15$ \\
\hline NCT01898611 & Completed & Frailty syndrome & Ghrelin & 01-07-13 & $31-08-16$ \\
\hline NCT01989793 & Completed & Sarcopenia & Losartan & $01-07-13$ & $01-10-16$ \\
\hline NCT01886196 & Completed & $\begin{array}{l}\text { Sarcopenia } \\
\text { Osteoporosis }\end{array}$ & Ibuprofen & 01-04-13 & 01-07-14 \\
\hline NCT02327091 & Completed & Muscle weakness & Alfacalcidol & 01-04-12 & $01-12-12$ \\
\hline NCT01666522 & Completed & Sarcopenia & Vitamin D & 01-04-11 & 01-09-11 \\
\hline NCT02305069 & Completed & $\begin{array}{l}\text { Obesity } \\
\text { Sarcopenia }\end{array}$ & $\begin{array}{l}\text { Pioglitazone } \\
\text { Insulin } \\
\text { Octreotide }\end{array}$ & 01-10-09 & 01-09-12 \\
\hline NCT00891696 & Completed & Sarcopenia & $\begin{array}{l}\text { Rapamycin } \\
\text { Sodium nitroprusside }\end{array}$ & 01-04-09 & 01-03-15 \\
\hline NCT00957801 & Completed & Sarcopenia & $\begin{array}{l}\text { Testosterone } \\
\text { Medrol }\end{array}$ & 01-03-09 & $01-12-15$ \\
\hline NCT00529659 & Completed & Sarcopenia & MK-0773 & 01-10-07 & 01-10-09 \\
\hline NCT00509405 & Completed & Healthy & Potassium citrate & 01-07-07 & $01-05-10$ \\
\hline NCT00475501 & Completed & $\begin{array}{l}\text { Male hypogonadism } \\
\text { Muscle atrophy } \\
\text { Sarcopenia } \\
\text { Benign prostate hypertrophy }\end{array}$ & $\begin{array}{l}\text { Testosterone enanthate } \\
\text { Finasteride }\end{array}$ & 01-01-07 & 01-10-14 \\
\hline NCT00315146 & Completed & $\begin{array}{l}\text { Obesity } \\
\text { Overweight with indications for } \\
\text { weight loss }\end{array}$ & Pioglitazone & 01-04-06 & 01-04-07 \\
\hline NCT00690534 & Completed & Sarcopenia & $\begin{array}{l}\text { Insulin regular } \\
\text { L-NMMA } \\
\text { Sodium nitroprusside }\end{array}$ & 01-09-05 & 01-08-12 \\
\hline NCT00128115 & Terminated & Hip Fracture & MK0677 & 01-09-05 & 01-08-07 \\
\hline NCT00240981 & Terminated & $\begin{array}{l}\text { Sarcopenia } \\
\text { Hypogonadism } \\
\text { Muscular diseases }\end{array}$ & Testosterone & 01-01-05 & 01-12-09 \\
\hline NCT00190060 & Completed & $\begin{array}{l}\text { Frailty } \\
\text { Sarcopenia }\end{array}$ & Testosterone & 01-10-04 & $31-12-08$ \\
\hline NCT00104572 & Completed & $\begin{array}{l}\text { Hypogonadism } \\
\text { Diabetes } \\
\text { Sarcopenia } \\
\text { Sarcopenia } \\
\text { Depression }\end{array}$ & $\begin{array}{l}\text { Androgel (Testosterone Gel) } \\
\text { Anastrozole (aromatase inhibitor) }\end{array}$ & 01-03-04 & $01-01-15$ \\
\hline NCT00183040 & Completed & $\begin{array}{l}\text { Sarcopenia } \\
\text { Muscle weakness } \\
\text { Frailty }\end{array}$ & $\begin{array}{l}\text { Testosterone } \\
\text { Recombinant human growth } \\
\text { hormone }\end{array}$ & 01-09-02 & 01-02-07 \\
\hline NCT00205686 & Completed & Healthy volunteers & DHEA & 01-04-01 & 01-09-05 \\
\hline NCT00474279 & Completed & Aging & MK-677 & 01-07-98 & 01-06-04 \\
\hline NCT00254371 & Completed & $\begin{array}{l}\text { Aging } \\
\text { Low DHEA for women } \\
\text { Low testosterone and DHEA for men }\end{array}$ & Androgen replacement therapy & 01-07-98 & 01-02-07 \\
\hline
\end{tabular}

Manual selection of interventional trials studying pharmacological products 


\section{References}

1. Reginster J-Y, Cooper C, Rizzoli R et al (2016) Recommendations for the conduct of clinical trials for drugs to treat or prevent sarcopenia. Aging Clin Exp Res 28:47-58. https://doi. org/10.1007/s40520-015-0517-y

2. Cruz-Jentoft AJ, Bahat G, Bauer J et al (2019) Sarcopenia: revised European consensus on definition and diagnosis. Age Ageing 48:16-31. https://doi.org/10.1093/ageing/afy169

3. Kilsby AJ, Sayer AA, Witham MD (2017) Selecting potential pharmacological interventions in sarcopenia. Drugs Aging 34:233-240. https://doi.org/10.1007/s40266-017-0444-z

4. Peña Ordóñez GG, Bustamante Montes LP, Ramírez Duran N et al (2017) Populations and outcome measures used in ongoing research in sarcopenia. Aging Clin Exp Res 29:695-700. https ://doi.org/10.1007/s40520-016-0610-x

5. Chen LK, Woo J, Assantachai P et al (2020) Asian working group for sarcopenia: 2019 consensus update on sarcopenia diagnosis and treatment. J Am Med Dir Assoc 21:300-307.e2. https://doi.org/10.1016/j.jamda.2019.12.012

6. Bhasin S, Travison TG, Manini TM et al (2020) Sarcopenia definition: the position statements of the sarcopenia definition and outcomes consortium. J Am Geriatr Soc. https://doi. org/10.1111/jgs.16372

7. Saggini R, Carmignano S, Cosenza L et al (2017) Sarcopenia in chronic illness and rehabilitative approaches. Frailty Sarcopenia Onset, Dev Clin Chall. https://doi.org/10.1016/j.colsu rfa.2011.12.014

8. Anker SD, Morley JE, von Haehling S (2016) Welcome to the ICD-10 code for sarcopenia. J Cachexia Sarcopenia Muscle 7:512-514. https://doi.org/10.1002/jcsm.12147

9. Vellas B, Fielding RA, Bens C et al (2018) Implications of ICD10 for sarcopenia clinical practice and clinical trials: report by the international conference on frailty and sarcopenia research task force. J Frailty Aging 7:2-9. https://doi.org/10.14283/ jfa. 2017.30

10. Hardee JP, Lynch GS (2019) Current pharmacotherapies for sarcopenia. Expert Opin Pharmacother 20:1645-1657. https:// doi.org/10.1080/14656566.2019.1622093

11. Morley JE (2016) Pharmacologic options for the treatment of sarcopenia. Calcif Tissue Int 98:319-333. https://doi.org/10.1007/ s00223-015-0022-5

12. Rooks D, Roubenoff R (2019) Development of pharmacotherapies for the treatment of sarcopenia. J Frailty Aging 8:120-130. https ://doi.org/10.14283/jfa.2019.11

13. Chumlea WC, Cesari M, Evans WJ et al (2011) Sarcopenia: designing phase IIB trials. J Nutr Health Aging 15:450-455

14. Cruz-Jentoft AJ, Landi F, Schneider SM et al (2014) Prevalence of and interventions for sarcopenia in ageing adults: a systematic review. Report of the International Sarcopenia Initiative (EWGSOP and IWGS). Age Ageing 43:748-759. https://doi. org/10.1093/ageing/afu115

15. Abellan Van Kan G, Cameron Chumlea W, Gillette-Guyonet S et al (2011) Clinical trials on sarcopenia: methodological issues regarding phase 3 trials NIH Public Access. Clin Geriatr Med 27:471-482. https://doi.org/10.1016/j.cger.2011.03.010

16. Visser M, Goodpaster BH, Kritchevsky SB et al (2005) Muscle mass, muscle strength, and muscle fat infiltration as predictors of incident mobility limitations in well-functioning older persons. J Gerontol A Biol Sci Med Sci 60:324-333

17. Landi F, Cesari M, Calvani R et al (2017) The "sarcopenia and physical fRailty IN older people: multi-componenT Treatment strategies" (SPRINTT) randomized controlled trial: design and methods. Aging Clin Exp Res 29:89-100. https://doi.org/10.1007/ s40520-016-0715-2
18. Pahor M, Guralnik JM, Ambrosius WT et al (2014) Effect of structured physical activity on prevention of major mobility disability in older adults: the LIFE study randomized clinical trial. JAMA 311:2387-2396. https://doi.org/10.1001/jama.2014.5616

19. Beaudart C, Rolland Y, Cruz-Jentoft AJ et al (2019) Assessment of muscle function and physical performance in daily clinical practice: a position paper endorsed by the european society for clinical and economic aspects of osteoporosis, osteoarthritis and musculoskeletal diseases (ESCEO). Calcif Tissue Int 105:1-14. https://doi.org/10.1007/s00223-019-00545-w

20. Schaap LA, Fox B, Henwood T et al (2016) Grip strength measurement: towards a standardized approach in sarcopenia research and practice. Eur Geriatr Med. https://doi.org/10.1016/j.eurge r.2015.11.012

21. Dodds RM, Syddall HHE, Cooper R et al (2014) Grip strength across the life course: normative data from twelve British studies. PLoS ONE 9:e113637. https://doi.org/10.1371/journ al.pone. 0113637

22. Cesari M, Kritchevsky SB, Newman AB et al (2009) Added value of physical performance measures in predicting adverse healthrelated events: results from the health, aging and body composition study. J Am Geriatr Soc 57:251-259. https://doi.org/10.111 1/j.1532-5415.2008.02126.x

23. Pavasini R, Guralnik J, Brown JC et al (2016) Short physical performance battery and all-cause mortality: systematic review and meta-analysis. BMC Med 14:215. https://doi.org/10.1186/s1291 6-016-0763-7

24. Studenski S, Perera S, Patel K et al (2011) Gait speed and survival in older adults. JAMA 305:50-58. https://doi.org/10.1001/ jama.2010.1923

25. Bischoff HA, Stähelin HB, Monsch AU et al (2003) Identifying a cut-off point for normal mobility: a comparison of the timed "up and go" test in community-dwelling and institutionalised elderly women. Age Ageing 32:315-320. https://doi.org/10.1093/agein $\mathrm{g} / 32.3 .315$

26. Guigoz Y, Vellas B, Garry PJ (1996) Assessing the nutritional status of the elderly: the mini nutritional assessment as part of the geriatric evaluation. Nutr Rev 54:S59-65

27. Cederholm T, Jensen GL (2017) To create a consensus on malnutrition diagnostic criteria: a report from the global leadership initiative on malnutrition (GLIM) meeting at the ESPEN Congress 2016. Clin Nutr 36:7-10. https://doi.org/10.1016/j. clnu.2016.12.001

28. Cederholm T, Jensen GL, Correia MITD et al (2019) GLIM criteria for the diagnosis of malnutrition - a consensus report from the global clinical nutrition community. J Cachexia Sarcopenia Muscle 38:1-9. https://doi.org/10.1016/j.clnu.2018.08.002

29. Sánchez-Rodríguez D, Marco E, Schott A-M et al (2018) Malnutrition according to ESPEN definition predicts long-term mortality in general older population: findings from the EPIDOS study-Toulouse cohort. Clin Nutr. https://doi.org/10.1016/j. clnu.2018.11.016

30. Beaudart C, Sanchez-Rodriguez D, Locquet M et al (2019) Malnutrition as a strong predictor of the onset of sarcopenia. Nutrients 11:2883. https://doi.org/10.3390/nu11122883

31. Delanaye P, Bataille S, Quinonez K et al (2019) Myostatin and insulin-like growth factor 1 are biomarkers of muscle strength, muscle mass, and mortality in patients on hemodialysis. J Ren Nutr 29:511-520. https://doi.org/10.1053/j.jrn.2018.11.010

32. Beaudart C, McCloskey E, Bruyère $O$ et al (2016) Sarcopenia in daily practice: assessment and management. BMC Geriatr 16:170. https://doi.org/10.1186/s12877-016-0349-4

33. Veronese N, Demurtas J, Soysal P et al (2019) Sarcopenia and health-related outcomes: an umbrella review of observational studies. Eur Geriatr Med 10:853-862. https://doi.org/10.1007/ s41999-019-00233-w 
34. de Wit M, Cooper C, Reginster JY (2019) Practical guidance for patient-centred health research. Lancet 393:1095-1096. https:// doi.org/10.1016/S0140-6736(19)30034-0

35. de Wit M, Cooper C, Tugwell P et al (2019) Practical guidance for engaging patients in health research, treatment guidelines and regulatory processes: results of an expert group meeting organized by the World Health Organization (WHO) and the European society for clinical and economic aspects of Osteoporosis, Osteoarthritis and Musculoskeletal Diseases (ESCEO). Aging Clin Exp Res. https://doi.org/10.1007/s40520-019-01193-8

36. Mercieca-Bebber R, King MT, Calvert MJ et al (2018) The importance of patient-reported outcomes in clinical trials and strategies for future optimization. Patient Relat Outcome Meas 9:353-367. https://doi.org/10.2147/prom.s156279

37. Qian HL (2009) Evaluating co-primary endpoints collectively in clinical trials. Biom J 51:137-145. https://doi.org/10.1002/ bimj.200710497

38. McLeod C, Norman R, Litton E et al (2019) Choosing primary endpoints for clinical trials of health care interventions. Contemp Clin Trials Commun 16:100486. https://doi.org/10.1016/j.conct c. 2019.100486

39. Tsekoura M, Billis E, Tsepis E et al (2018) The effects of group and home-based exercise programs in elderly with sarcopenia: a randomized controlled trial. J Clin Med 7:480. https://doi. org/10.3390/jcm7120480

40. Geerinck A, Bruyere O, Locquet M et al (2018) Evaluation of the Responsiveness of the SarQoL((R)) Questionnaire, a patientreported outcome measure specific to sarcopenia. Adv Ther 35:1842-1858. https://doi.org/10.1007/s12325-018-0820-z

41. Kwon S, Perera S, Pahor M et al (2009) What is a meaningful change in physical performance? Findings from a clinical trial in older adults (The LIFE-P study). J Nutr Heal Aging 13:538-544. https://doi.org/10.1007/s12603-009-0104-Z

42. Vestergaard S, Patel KV, Bandinelli S et al (2009) Characteristics of 400-meter walk test performance and subsequent mortality in older adults. Rejuvenation Res 12:177-184. https://doi. org/10.1089/rej.2009.0853

43. Newman AB, Simonsick EM, Naydeck BL et al (2006) Association of long-distance corridor walk performance with mortality, cardiovascular disease, mobility limitation, and disability. JAMA 295:2018-2026. https://doi.org/10.1001/jama.295.17.2018

44. Hardy SE, Kang Y, Studenski SA, Degenholtz HB (2011) Ability to walk $1 / 4$ mile predicts subsequent disability, mortality, and health care costs. J Gen Intern Med 26:130-135. https:// doi.org/10.1007/s11606-010-1543-2

45. Vasunilashorn S, Coppin AK, Patel KV et al (2009) Use of the short physical performance battery score to predict loss of ability to walk 400 meters: analysis from the InCHIANTI study. J Gerontol Ser A Biol Sci Med Sci 64A:223-229. https://doi. org/10.1093/gerona/gln022

46. Cesari M, Onder G, Zamboni V et al (2008) Physical function and self-rated health status as predictors of mortality: results from longitudinal analysis in the ilSIRENTE study. BMC Geriatr 8:34. https://doi.org/10.1186/1471-2318-8-34

47. Hedman S, Nydahl M, Faxén-Irving G (2016) Individually prescribed diet is fundamental to optimize nutritional treatment in geriatric patients. Clin Nutr 35:692-698. https://doi. org/10.1016/j.clnu.2015.04.018

48. Hiligsmann $\mathrm{M}$, Beaudart $\mathrm{C}$, Bruyère $\mathrm{O}$ et al (2019) Outcome priorities for older persons with sarcopenia. J Am Med Dir Assoc. https://doi.org/10.1016/j.jamda.2019.08.026

49. Evans CJ, Chiou C-F, Fitzgerald KA et al (2011) Development of a new patient-reported outcome measure in sarcopenia. $\mathrm{J}$ Am Med Dir Assoc 12:226-233. https://doi.org/10.1016/j.jamda 2010.09.010
50. Beaudart C, Biver E, Reginster J-Y et al (2015) Development of a self-administrated quality of life questionnaire for sarcopenia in elderly subjects: the SarQoL. Age Ageing 44:960-966. https ://doi.org/10.1093/ageing/afv133

51. Beaudart C, Biver E, Reginster J-Y et al (2017) Validation of the SarQoL®, a specific health-related quality of life questionnaire for Sarcopenia. J Cachexia Sarcopenia Muscle. https://doi. org/10.1002/jcsm.12149

52. Geerinck A, Alekna V, Beaudart C et al (2019) Standard error of measurement and smallest detectable change of the sarcopenia quality of Life (Sarqol) questionnaire: an analysis of subjects from 9 validation studies. PLoS ONE 14:e0216065. https://doi. org/10.1371/journal.pone.0216065

53. Ader DN (2007) Developing the patient-reported outcomes measurement information system (PROMIS). Med Care 45:1-2. https://doi.org/10.1097/01.mlr.0000260537.45076.74

54. Zeeshan B, David C, Jensen S, Shaunfield S Development of a context of use and PROMIS Physical Function outcome assessment for patients with sarcopenia.

55. Bodilsen AC, Juul-Larsen HG, Petersen J et al (2015) Feasibility and inter-rater reliability of physical performance measures in acutely admitted older medical patients. PLoS ONE 10:e0118248. https://doi.org/10.1371/journal.pone.0118248

56. Roberts HC, Denison HJ, Martin HJ et al (2011) A review of the measurement of grip strength in clinical and epidemiological studies: towards a standardised approach. Age Ageing 40:423429. https://doi.org/10.1093/ageing/afr051

57. Beaudart C, Reginster JY, Slomian J et al (2015) Estimation of sarcopenia prevalence using various assessment tools. Exp Gerontol. https://doi.org/10.1016/j.exger.2014.11.014

58. Bohannon RW (2017) Test-Retest reliability of measurements of hand-grip strength obtained by dynamometry from older adults: a systematic review of research in the PubMed Database. J Frailty Aging 6:83-87. https://doi.org/10.14283/jfa.2017.8

59. Santanasto AJ, Glynn NW, Lovato LC et al (2017) Effect of Physical activity versus health education on physical function, grip strength and mobility. J Am Geriatr Soc 65:1427-1433. https://doi.org/10.1111/jgs.14804

60. Nitschke JE, McMeeken JM, Burry HC, Matyas TA (1999) When is a change a genuine change?: a clinically meaningful interpretation of grip strength measurements in healthy and disabled women. J Hand Ther 12:25-30. https://doi.org/10.1016/ S0894-1130(99)80030-1

61. Beaudart C, Dawson A, Shaw SC et al (2017) Nutrition and physical activity in the prevention and treatment of sarcopenia: systematic review. Osteoporos Int. https://doi.org/10.1007/s0019 8-017-3980-9

62. Buckinx F, Landi F, Cesari M et al (2018) Pitfalls in the measurement of muscle mass: a need for a reference standard. J Cachexia Sarcopenia Muscle 9:269-278. https://doi.org/10.1002/ jcsm. 12268

63. Dehghan M, Merchant AT (2008) Is bioelectrical impedance accurate for use in large epidemiological studies? Nutr J 7:26. https:// doi.org/10.1186/1475-2891-7-26

64. Jensen SM, Mølgaard C, Ejlerskov KT et al (2015) Validity of anthropometric measurements to assess body composition, including muscle mass, in 3-year-old children from the SKOT cohort. Matern Child Nutr 11:398-408. https://doi.org/10.1111/ mcn. 12013

65. Evans WJ, Hellerstein M, Orwoll E et al (2019) D 3 -Creatine dilution and the importance of accuracy in the assessment of skeletal muscle mass. J Cachexia Sarcopenia Muscle 10:14-21. https ://doi.org/10.1002/jcsm. 12390

66. Perkisas S, Baudry S, Bauer J et al (2018) Application of ultrasound for muscle assessment in sarcopenia: towards standardized 
measurements. Eur Geriatr Med 9:739-757. https://doi. org/10.1007/s41999-018-0104-9

67. Auyeung TW, Lee SW, Leung J et al (2014) Age-associated decline of muscle mass, grip strength and gait speed: a 4-year longitudinal study of 3018 Community-Dwelling Older Chinese. Geriatr Gerontol Int. https://doi.org/10.1111/GGI.12213

68. Shaw SC, Dennison EM, Cooper C (2017) Epidemiology of sarcopenia: determinants throughout the lifecourse. Calcif Tissue Int 101:229-247. https://doi.org/10.1007/s00223-017-0277-0

69. Haran PH, Rivas DA, Fielding RA (2012) Role and potential mechanisms of anabolic resistance in sarcopenia. J Cachexia Sarcopenia Muscle 3:157. https://doi.org/10.1007/S1353 9-012-0068-4

70. Verlaan S, Aspray TJ, Bauer JM et al (2017) Nutritional status, body composition, and quality of life in community-dwelling sarcopenic and non-sarcopenic older adults: a case-control study. Clin Nutr 36:267-274. https://doi.org/10.1016/J.CLNU.2015.11.013

71. Rizzoli R, Stevenson JC, Bauer JM et al (2014) The role of dietary protein and vitamin D in maintaining musculoskeletal health in postmenopausal women: a consensus statement from the European society for clinical and economic aspects of osteoporosis and osteoarthritis (ESCEO). Maturitas 79:122-132. https://doi. org/10.1016/j.maturitas.2014.07.005

72. Paddon-Jones D, Rasmussen BB (2009) Dietary protein recommendations and the prevention of sarcopenia. Curr Opin Clin Nutr Metab Care 12:86-90. https://doi.org/10.1097/MCO.0b013e3283 1 cef8b

73. Tieland M, van de Rest O, Dirks ML et al (2012) Protein supplementation improves physical performance in frail elderly people: a randomized, double-blind, placebo-controlled trial. J Am Med Dir Assoc 13:720-726. https://doi.org/10.1016/j.jamda.2012.07.005

74. Bauer JM, Mikušová L, Verlaan S et al (2020) Safety and tolerability of 6-month supplementation with a vitamin D, calcium and leucine-enriched whey protein medical nutrition drink in sarcopenic older adults. Aging Clin Exp Res. https://doi.org/10.1007/ s40520-020-01519-x

75. Beaudart C, Locquet M, Touvier M et al (2019) Association between dietary nutrient intake and sarcopenia in the SarcoPhAge study. Aging Clin Exp Res. https://doi.org/10.1007/s40520-01901186-7

76. Sanz-París A, Gómez-Candela C, Martín-Palmero Á et al (2016) Application of the new ESPEN definition of malnutrition in geriatric diabetic patients during hospitalization: a multicentric study. Clin Nutr 35:1564-1567. https://doi.org/10.1016/j. clnu.2016.02.018

77. Peterson MD, Rhea MR, Sen A, Gordon PM (2010) Resistance exercise for muscular strength in older adults: a metaanalysis. Ageing Res Rev 9:226-237. https://doi.org/10.1016/j. arr.2010.03.004

78. Beckwée D, Delaere A, Aelbrecht S et al (2019) Exercise interventions for the prevention and treatment of sarcopenia. A systematic umbrella review. J Nutr Heal Aging 23:494-502. https://doi. org/10.1007/s12603-019-1196-8

79. Meier NF, Lee DC (2019) Physical activity and sarcopenia in older adults. Aging Clin Exp Res. https://doi.org/10.1007/s4052 0-019-01371-8

80. Xia W, Cooper C, Li M et al (2019) East meets West: current practices and policies in the management of musculoskeletal aging. Aging Clin Exp Res 31:1351-1373. https://doi.org/10.1007/s4052 0-019-01282-8

81. Taylor HL, Jacobs DR, Schucker B et al (1978) A questionnaire for the assessment of leisure time physical activities. J Chronic Dis 31:741-755. https://doi.org/10.1016/0021-9681(78)90058-9

82. Logan SL, Gottlieb BH, Maitl SB et al (2013) The physical activity scale for the elderly (PASE) questionnaire; does it predict physical health? Int J Environ Res Public Health 10:3967-3986. https://doi.org/10.3390/ijerph10093967

83. Beuscart J-B, Knol W, Cullinan S et al (2018) International core outcome set for clinical trials of medication review in multi-morbid older patients with polypharmacy. BMC Med 16:21. https:// doi.org/10.1186/s12916-018-1007-9

Publisher's Note Springer Nature remains neutral with regard to jurisdictional claims in published maps and institutional affiliations.

\section{Affiliations}

\section{Jean-Yves Reginster ${ }^{1} \cdot$ Charlotte Beaudart $^{1}$ (D) Nasser Al-Daghri ${ }^{2} \cdot$ Bernard Avouac $^{3}$. Jürgen Bauer ${ }^{4}$. Nathalie Bere ${ }^{5}$. Olivier Bruyère ${ }^{1}$. Francesca Cerreta ${ }^{5} \cdot$ Matteo Cesari $^{6,7} \cdot$ Mario Miguel Rosa $^{8} \cdot$ Cyrus Cooper $^{9}$. Alfonso J. Cruz Jentoft ${ }^{10}$. Elaine Dennison ${ }^{9}$. Anton Geerinck ${ }^{1}$. Evelien Gielen ${ }^{11,24}$. Francesco Landi ${ }^{12}$. Andrea Laslop ${ }^{13}$. Stefania Maggi ${ }^{14}$. María Concepción Prieto Yerro ${ }^{15} \cdot$ René Rizzoli $^{16}$. Hildrun Sundseth ${ }^{17}$. Cornel Sieber ${ }^{18,25} \cdot$ Andrea Trombetti $^{16} \cdot$ Bruno Vellas $^{19} \cdot$ Nicola Veronese $^{20} \cdot$ Marjolein Visser $^{21} \cdot$ Mila Vlaskovska $^{22}$. Roger A. Fielding ${ }^{23}$}

1 WHO Collaborating Center for Public Health Aspects of Musculo-Skeletal Health and Ageing, Division of Public Health, Epidemiology and Health Economics, University of Liège, Avenue Hippocrate 13, CHU B23, 4000 Liege, Belgium

2 Chair for Biomarkers of Chronic Diseases, Biochemistry Department, College of Science, King Saud University, Riyadh 11451, Saudi Arabia

3 Department of Rheumatology, CHU Henri Mondor, Creteil, France

4 Center for Geriatric Medicine and Network Aging Research, Heidelberg University, Heidelberg, Germany
5 European Medicines Agency, Amsterdam, The Netherlands

6 Department of Clinical Sciences and Community Health, University of Milan, Milan, Italy

7 Geriatric Unit, Fondazione IRCCS Ca' Granda Ospedale Maggiore Policlinico, Milan, Italy

8 Departamento de Neurociências/Laboratório de Farmacologia Clínica E Terapêutica, Faculdade de Medicina da Universidade de Lisboa, Lisbon, Portugal

9 MRC Lifecourse Epidemiology Unit, University of Southampton, Southampton, UK 
10 Hospital Universitario Ramón Y Cajal (IRYCIS), Madrid, Spain

11 Division of Geriatrics, UZ Leuven, Leuven, Belgium

12 Department of Geriatrics, Neurosciences and Orthopedics, Catholic University of the Sacred Heart Rome, Milan, Italy

13 Scientific Office, Federal Office for Safety in Health Care, Vienna, Austria

14 CNR Aging Branch-IN, Padua, Italy

15 Agencia Española de Medicamentos Y Productos Sanitarios, Madrid, Spain

16 Division of Bone Diseases, Geneva University Hospitals and Faculty of Medicine, Geneva, Switzerland

17 European Institute of Women's Health, Dublin, Ireland

18 Institute for Biomedicine of Aging Friedrich-Alexander University Erlangen-Nürnberg, Erlangen, Germany
19 Department of Internal and Geriatrics Medicine, Gerontopole, CHU de Toulouse, UMR 1027 INSERM, University Toulouse III, Toulouse, France

20 Department of Internal Medicine, Geriatrics Section, University of Palermo, Palermo, Italy

21 Department of Health Sciences, Vrije Universiteit Amsterdam, Amsterdam, The Netherlands

22 Medical Faculty, Department of Pharmacology and Toxicology, Medical University Sofia, Sofia, Bulgaria

23 Nutrition, Exercise Physiology and Sarcopenia Laboratory, Jean Mayer USDA Human Nutrition Research Center On Aging, Tufts University, Boston, USA

24 Gerontology and Geriatrics, Department of Public Health and Primary Care, KU Leuven, Leuven, Belgium

25 Kantonsspital Winterthur, Winterthur, Switzerland 